\title{
Using Enstrophy-Based Diagnostics in an Ensemble for Two Blocking Events
}

\author{
Andrew D. Jensen and Anthony R. Lupo \\ Department of Soil, Environmental, and Atmospheric Science, University of Missouri, 302 Anheuser Busch Natural Resources Building, \\ Columbia, MO 65211, USA \\ Correspondence should be addressed to Andrew D. Jensen; jensenad@missouri.edu
}

Received 10 September 2013; Revised 4 November 2013; Accepted 8 November 2013

Academic Editor: Yafei Wang

Copyright (C) 2013 A. D. Jensen and A. R. Lupo. This is an open access article distributed under the Creative Commons Attribution License, which permits unrestricted use, distribution, and reproduction in any medium, provided the original work is properly cited.

\begin{abstract}
Recent research has used enstrophy-based diagnostics to identify the development and dissipation stages of blocking events. These previous studies made use of reanalysis data sets in the calculations of the enstrophy-based diagnostics, such as the NCEP-NCAR reanalysis $\left(2.5^{\circ} \times 2.5^{\circ}\right)$ of geopotential height and horizontal winds. However, none of these studies has explored the use of the enstrophy-based diagnostics in weather or climate models with higher horizontal resolution. In this paper, the enstrophy-based diagnostics are used to analyze two blocking events, using data from the ERA-Interim reanalysis data set $\left(0.75^{\circ} \times 0.75^{\circ}\right)$ and also the Global Ensemble Forecast System (GEFS) $\left(1^{\circ} \times 1^{\circ}\right)$. The results of this work indicate that using an ensemble may be more effective than a single dynamical control forecast in evaluating the enstrophy-based diagnostic quantities, and that the results are similar to those obtained with coarser resolution.
\end{abstract}

\section{Introduction}

Many studies have noted an upscale cascade of enstrophy upstream of blocking events (see, e.g., [1, 2]). Moreover, in [3], enstrophy and large-scale instability are compared by means of finite-time Lyapunov exponents. Using these ideas and the instability at block onset and decay [4], in a series of recent articles (see [5-8]), enstrophy-based diagnostics have been used to study large-scale stability changes during the development and termination of blocking events. These studies used reanalysis data sets such as the NCEP-NCAR reanalysis of geopotential heights and winds to calculate the enstrophybased diagnostics. However, no work has yet explored the use of these diagnostics in weather or climate models or in an ensemble.

The utility of using ensemble-based forecasting to better predict blocking is well known (e.g., [9-11]). Several studies note the increased skill of forecasts of blocking episodes over solely dynamical prediction methods. For example, [10] showed that ECMWF ensemble prediction system forecasts of blocking are more skilful than the deterministic and climatology forecasts of Euro-Atlantic sector blocking, although blocking onset was better predicted than block decay overall. In [9], it was found that ensemble forecasts which were calibrated to correct for the under prediction of blocking were more accurate than uncalibrated ensemble forecasts. [11] found the ensemble mean to perform better than the control group for forecast times longer than 3-4 days in two atmospheric models. Errors were found to be largest at block onset and decay (see also [4]).

The purpose of this study is to use the enstrophy-based diagnostics (explained below and introduced in $[6,7]$ ) to analyze two blocking events, using data from the ERA-Interim reanalysis and also the Global Ensemble Forecast System (GEFS), both of which have higher horizontal resolution than the NCEP-NCAR reanalysis data set. The previous results in this area used relatively low-resolution $\left(2.5^{\circ} \times 2.5^{\circ}\right)$ data in the calculations. Thus, a primary objective of this study is to determine if the results are sensitive to the resolution used in the calculations by employing model data with higher horizontal resolution and thus to assess the extension of the overall usefulness of the diagnostics. The outcomes suggest that the use of an ensemble is preferable over a single 
dynamical control forecast to the use of the enstrophy-based diagnostics in a weather model.

The outline of the paper is as follows. In Section 2, the data sets and the enstrophy-based diagnostics and their use are explained. In Section 3, two blocking episodes are studied by means of these diagnostics using ERA-Interim reanalysis data and GEFS. In Section 4, we discuss and summarize our conclusions.

\section{Data and Methods}

2.1. Preliminaries. In order to explain the enstrophy diagnostics to be used, the local Lyapunov exponents for the barotropic vorticity equation must first be considered. Local Lyapunov exponents for the barotropic vorticity equation, where $\zeta$ is relative vorticity, are defined by $\lambda_{i}\left(\zeta_{0}, T\right)=$ $(1 / 2 n) \log \nu_{i}$ for initial $\zeta_{0}$ and time $T=n \Delta t$. The $\nu_{i}$ are the eigenvalues of $M_{n}^{*} M_{n}$, where $M=\prod_{k=-n}^{k=n} A(k \Delta t)$ and $A(t)$ is the linearization operator of the barotropic vorticity equation. Thus, the local Lyapunov exponents provide a measure of finite-time instability. In [12], finite-time instability is estimated by means of the largest singular value (eigenvalues of $M_{n}^{*} M_{n}$ ) in magnitude in a kinetic energy norm. Here, the approximation introduced in [3] is used as a measure of the finite-time stability.

The argument given in [3] proceeds as follows. A frictionless, nondivergent barotropic flow is assumed. As shown in [3], the results to be described are not fundamentally affected by orography. The barotropic vorticity equation can be written in terms of a stream function $\psi$ :

$$
\frac{\partial \nabla^{2} \psi}{\partial t}+J\left(\psi, \nabla^{2} \psi\right)=0
$$

where $\nabla^{2} \psi=\zeta$ and $\nabla^{2}$ is the Laplacian operator. Now, (1) can be linearized as follows:

$$
\frac{\partial \zeta^{\prime}}{\partial t}+A \zeta^{\prime}=0
$$

where $A$ is the linearization operator, $\psi=\bar{\psi}+\psi^{\prime}$, and $\nabla^{2} \psi^{\prime}=\zeta^{\prime}$. Using the Crank-Nicholson scheme, the following equation may be obtained:

$$
\frac{\zeta^{\prime}(t+\delta t)-\zeta^{\prime}(t)}{\delta t}+A\left(\bar{\psi}\left(t+\frac{\delta t}{2}\right)\right) \frac{\zeta^{\prime}(t+\delta t)+\zeta^{\prime}(t)}{2}=0 .
$$

To estimate the Lyapunov exponents, (3) can be rearranged, where $I$ is the identity operator:

$$
\begin{aligned}
\zeta^{\prime}(t+\delta t)= & {\left[I+\frac{\tau}{2} A\left(\bar{\psi}\left(t+\frac{\delta t}{2}\right)\right)\right]^{-1} } \\
& \times\left[I-\frac{\tau}{2} A\left(\bar{\psi}\left(t+\frac{\delta t}{2}\right)\right)\right] \zeta^{\prime}(t) .
\end{aligned}
$$

The operator in brackets in (4) is estimated by $S=A+A^{*}$, where $A^{*}$ is the adjoint operator defined for vectors $\mathbf{x}, \mathbf{y}$ and inner product $(\cdot, \cdot)$ by $(A \mathbf{x}, \mathbf{y})=\left(\mathbf{x}, A^{*} \mathbf{y}\right)$. To model blocked flow, suppose that

$$
\psi^{\prime}(x, y)=\psi(y) e^{i k x}
$$

When

$$
S=A+A^{*}=\bar{u} \frac{\partial}{\partial x} \nabla^{2}-\nabla^{2}\left(\bar{u} \frac{\partial}{\partial x}\right),
$$

it can be shown that $S=-i k K$, where $K$ is a skew-symmetric operator. Because the eigenvalues $S$ are symmetric about zero, the eigenvalues of the operator $K^{2}$ are studied instead. Using finite differencing to project onto finite space, the sum of the positive local Lyapunov exponents can be shown to be determined by the integral of enstrophy, where the integral is over a finite and bounded region.

2.2. Enstrophy Advection and Its Integral. As sketched above,

$$
\sum_{\lambda_{i}>0} \lambda_{i} \approx \int \zeta^{2} \mathrm{~d} A
$$

where the integral is taken over the Northern Hemisphere here. Since the $\lambda_{i}$ change with time, (7) may be differentiated to obtain

$$
\frac{\partial\left(\sum_{\lambda_{i}>0} \lambda_{i}\right)}{\partial t} \approx \frac{\partial}{\partial t} \int \zeta^{2} \mathrm{~d} A=-\int \mathbf{v} \cdot \nabla \zeta^{2} \mathrm{~d} A,
$$

where nondivergent, frictionless barotropic flow on an $f$ plane has been assumed.

To get a more accurate derivative, it is possible to proceed as in [13] and consider the barotropic vorticity equation in the form

$$
\begin{gathered}
\left(\frac{\partial}{\partial t}+u_{0} \frac{\partial}{\partial x}\right)\left(\nabla^{2} \psi-F \psi\right)+J\left(\psi, \nabla^{2} \psi+h\right) \\
+\left(\beta+F u_{0}\right) \frac{\partial \psi}{\partial x}+u_{0} \frac{\partial h}{\partial x}=-J\left(\psi^{\prime}, \nabla^{2} \psi^{\prime}\right)_{P}
\end{gathered}
$$

where $u_{0}$ represents the basic state westerly wind, $\psi$ and $\psi^{\prime}$ represent planetary and synoptic scales of the stream function, respectively; $h$ is a nondimensional topography term, $F=\left(L / R_{d}\right)^{2}$ where $R_{d}$ is the Rossby deformation radius, and the subscript " $P$ " represents the planetary scale component. However, in [3], the topography and friction were omitted to obtain (7), as described above. Hence, here we do not retain such terms in the derivative, while realizing that other terms can be included to make the result more accurate. Another reason for not retaining such terms is a practical one: contours of enstrophy advection are easy to interpret and are explained in detail in [6].

2.3. Methodology. The enstrophy-based diagnostics (described in detail above) to be used in the analysis of the two blocking events considered below were introduced in $[6,7]$, and they are as follows:

$$
\mathrm{IRE} \equiv \int \zeta^{2} \mathrm{~d} A
$$

$$
\operatorname{DIRE} \equiv-\int \mathbf{v} \cdot \nabla \zeta^{2} \mathrm{~d} A
$$


where the integral is evaluated over some finite area on an isobaric surface. The integrated enstrophy (IRE), or (10), has been shown to be related to finite-time instability by means of the finite-time Lyapunov exponents as described above. Peaks in (10) are therefore a measure of local maximum flow instability, in particular, planetary scale flow. In [7], it was observed that the IRE increased sharply at block onset, indicating an increase in planetary flow instability. The IRE was then observed to decrease to a local minimum and to increase again at block decay to a local maximum value. On the other hand, the DIRE, or (11), is the derivative of (10) assuming barotropic, inviscid flow, and increasing (decreasing) instability is indicated when (11) is positive (negative), while maxima in the IRE field can often be found (see [6]) when (11) crosses the time-axis from positive to negative.

The diagnostics (10) and (11) were calculated in a spherical coordinate system for the Northern Hemisphere using ECMWF ERA-Interim data, obtained from the ECMWF data server. The quantities used in this study are the zonal and meridional wind components, geopotential height, and relative vorticity with a $0.75^{\circ} \times 0.75^{\circ}$ horizontal resolution at $500 \mathrm{hPa}$. The same quantities were also used from the Global Ensemble Forecast System (GEFS), which has 20 members plus the ensemble mean and control with a horizontal resolution of $1^{\circ} \times 1^{\circ}$, in order to calculate (10) and (11). We chose representative ensemble members from the 20 , besides the ensemble mean and control, which are shown in the plots below.

The blocking definition used in this study is that of Lupo and Smith [14], which can be described as a synthesis of the subjective Rex criteria (see $[15,16]$ ) and the objective LejenasOkland criteria (see [17]), but in which a blocking event is defined to persist for at least five days. More specifically, the blocking criteria used here (i) must satisfy the Rex $[15,16]$ criteria for at least five days and (ii) must have a negative or small positive zonal index that can be identified on a time-longitude or Hovmöller diagram. (iii) Conditions (i) and (ii) must be satisfied for 24 hours after (before) onset (termination); (iv) the blocking event should be poleward of $35 \mathrm{~N}$ during its lifetime, and the ridge should have an amplitude of greater than $5^{\circ}$ latitude; and (v) blocking onset is defined to occur when condition (iv) and and one of the conditions (i) or (ii) is satisfied, while (vi) termination or decay is designated at the time the event fails to satisfy condition (v) for a 24-hour period or longer. This definition was used to detect the blocking onset and decay times for the events considered below.

\section{Dynamic Analysis}

3.1. Event 1: October 11-19, 2012. The first blocking event considered here occurred October 11-19, 2012 and was centered at $160 \mathrm{E}$ (see Figures 1, 2, and 3). The ERA-Interim reanalysis overall appeared to have tighter gradients than GEFS. The mean height contours of the GEFS ensemble mean and control appear to be similar, while differences with the ERA calculated mean heights are borne out in the calculations below.

The IRE (integrated enstrophy) and DIRE (derivative of IRE) for the ERA-Interim data were rescaled and are shown together in Figure 4. Two distinct maxima can be seen on

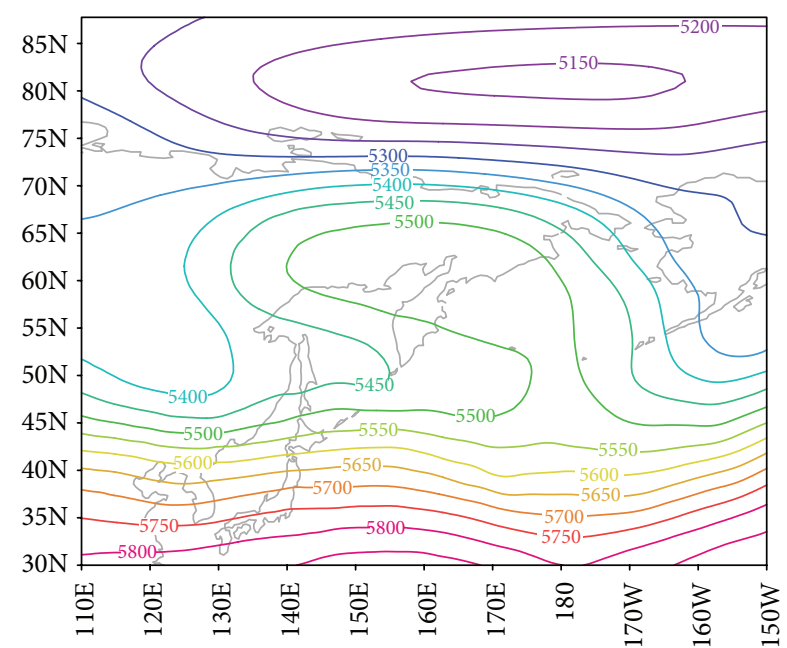

FIGURE 1: ERA-Interim reanalysis time-averaged geopotential heights for October 11-19, 2012.

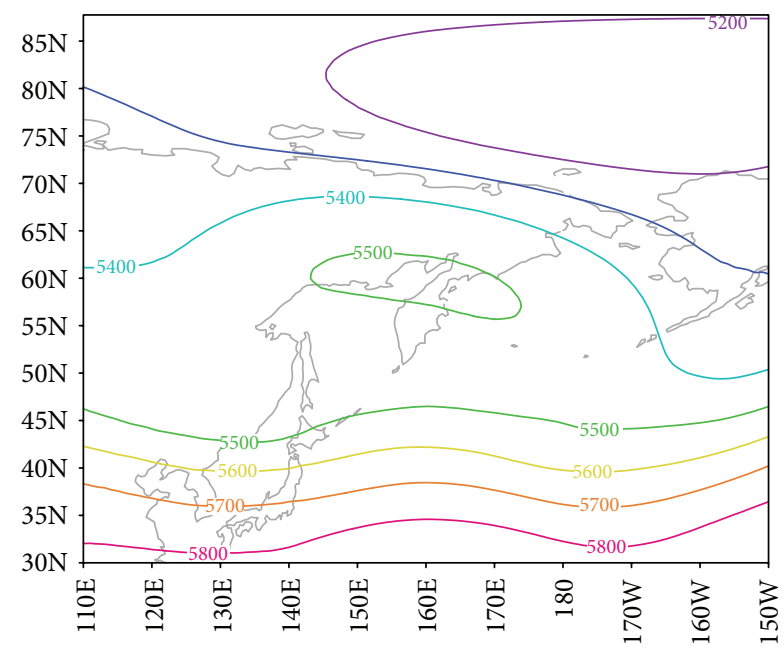

FIGURE 2: GEFS ensemble mean time-averaged geopotential heights for October 11-19, 2012.

the IRE time series, corresponding to block onset and decay, respectively. A clear upward trend can be seen in the IRE field during the block development stage. During the maintenance stage of the event, the IRE dips to a minimum value and again achieves a local maximum during the dissipation stage of the blocking event. On the other hand, the DIRE crosses the timeaxis from positive to negative at block onset, reflecting the local instability maximum. During the maintenance stage, the DIRE is negative until the 16th of October, reflecting decreasing instability. The DIRE then assumes positive values until it again crosses the time-axis from positive to negative which reflects the local instability maximum at block decay.

The IRE for the GEFS mean, control, and two representative ensemble members are plotted alongside the ERAInterim IRE for comparison (see Figure 5). The IRE for the GEFS mean is strictly decreasing, and the GEFS control appears to behave similarly. The ensemble member 11 is an 


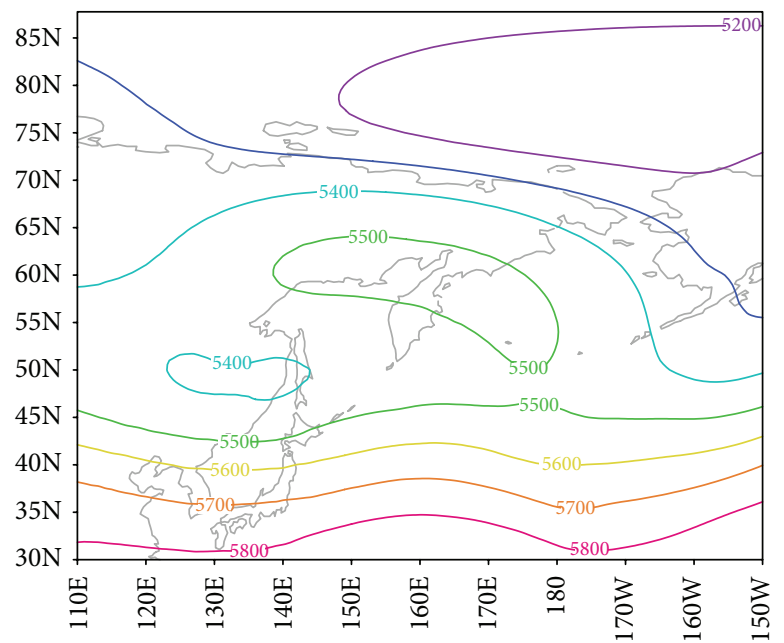

FIGURE 3: GEFS control time averaged geopotential heights for October 11-19, 2012.

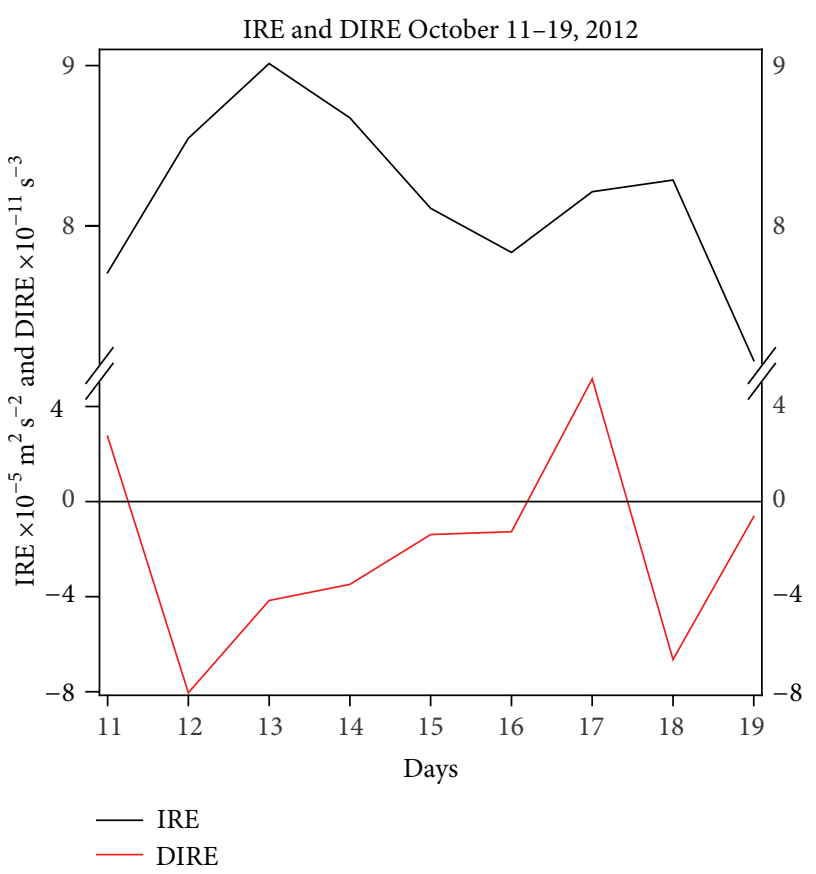

FIGURE 4: IRE and DIRE from ERA-Interim reanalysis for October $11-19,2012$.

extreme outlier and likely affected the poor performance of the ensemble mean. However, M1 and M2, which are members 5 and 15 of the ensemble and are representatives of other members of the ensemble, appear closer to the ERA-Interim IRE in that they reach maxima in the IRE field, if somewhat lagging in time. M2 also appears to reach a local maximum at block decay.

Now, the DIRE for the GEFS mean, control, and two ensemble members are plotted alongside the ERA-Interim DIRE for comparison (see Figure 6). In contradistinction to the GEFS IRE, all of the GEFS members plotted achieve a distinct local maximum in instability at block onset as

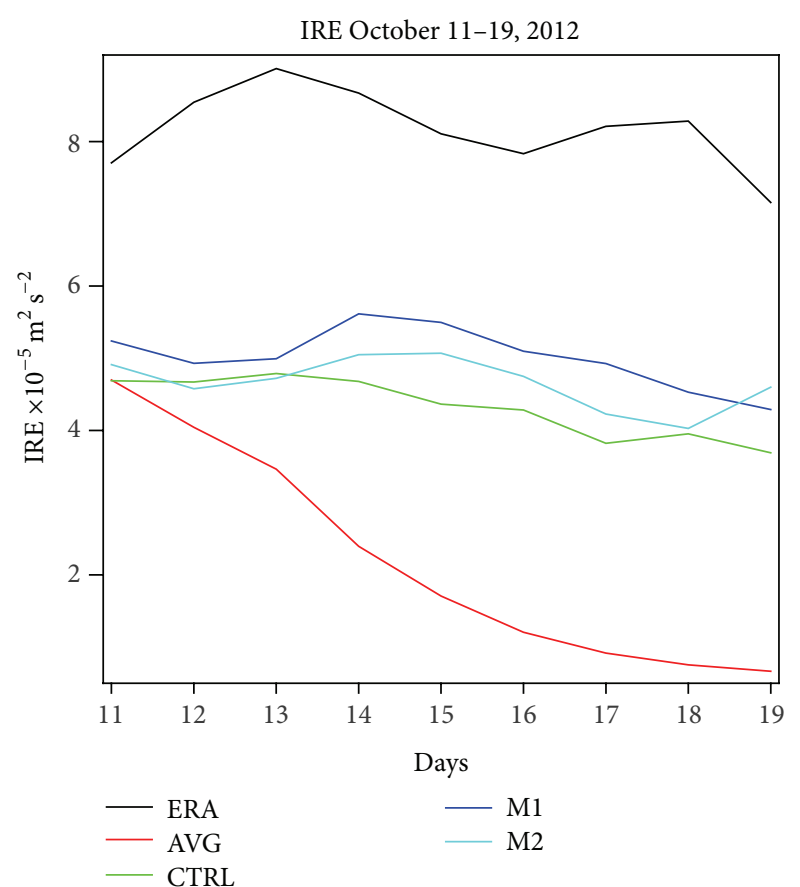

FIGURE 5: ERA and GEFS values of IRE for October 11-19, 2012. Shown are ERA reanalysis (black), GEFS ensemble mean (red), GEFS control (green), and two other ensemble members (dark and light blue).

reflected by their crossing the time-axis from positive to negative. Similar to the behavior of the IRE as calculated from GEFS, M1 and M2 from the ensemble show results that are similar to the ERA-Interim DIRE with the DIRE crossing the time-axis from positive to negative values for block onset and decay periods.

3.2. Event 2: March 9-14, 2013. The second event occurred March 9-14, 2013 and was centered at 170 W (see Figures 7, 8, and 9). In this case, ERA-Interim, GEFS mean, and GEFS control appear more similar to each other compared to the first case. However, the contours over Alaska and gradients over the Pacific are different for the reanalysis compared to GEFS.

Again, the IRE and DIRE for the ERA-Interim data were rescaled and are shown together in Figure 10. There, the IRE field is at a local maximum value during the block development stage. The IRE decreases to a minimum value around the 13th of March during the maintenance stage of the event. Finally, the IRE increases to a local maximum by the end of the event. Now, the DIRE crosses the time-axis from positive to negative values on the 9th of March, reflecting the local instability maximum at block onset. The instability decreases (DIRE is negative) during the maintenance stage until the 11th of March. The DIRE then takes on positive values until again crossing the time-axis from positive to negative values, which reflects the local instability maximum during the dissipation stage of the block, as expected. 


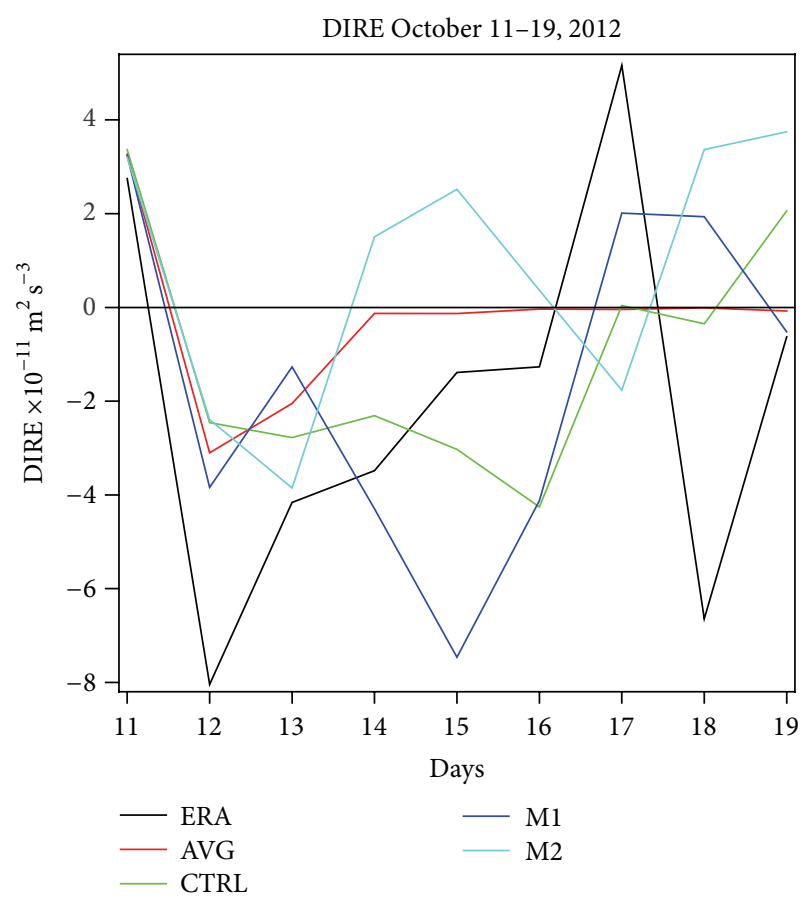

FIGURE 6: ERA and GEFS values of DIRE for October 11-19, 2012. Shown are ERA reanalysis (black), GEFS ensemble mean (red), GEFS control (green), and two other ensemble members (dark and light blue).

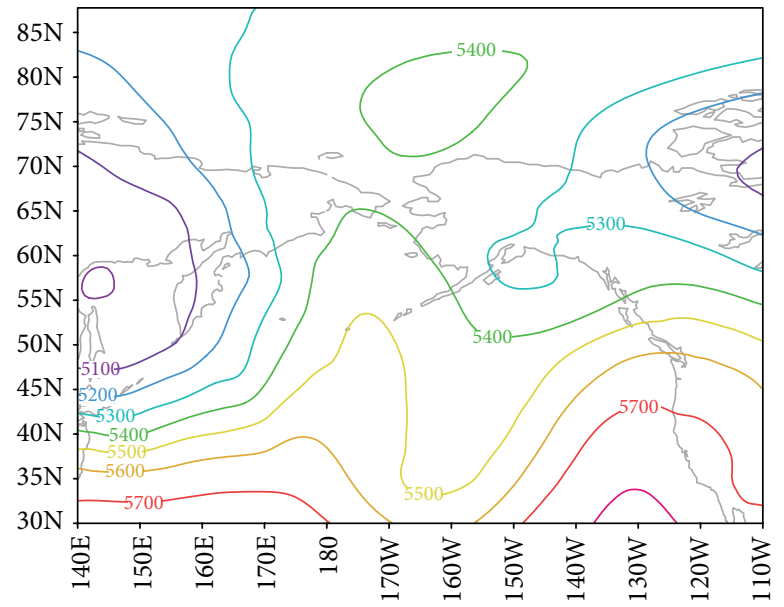

FIGURE 7: ERA-Interim reanalysis time-averaged geopotential heights for March 9-14, 2013.

Again, The IRE for the GEFS mean, control, and two ensemble members are plotted alongside the ERA-Interim IRE (see Figure 11). The IRE for the GEFS mean and control does not appear to reflect a realistic tendency in the IRE as seen in previous work [5-8]. The ensemble members 10 and 17 are extreme outliers and contributed to the poor performance of the ensemble mean. Again, M1 and M2, which are members 5 and 15 of the ensemble and are representatives of the other members, appear closer to the ERA-Interim IRE in that they both reach maxima (lagging in time again) in the IRE field

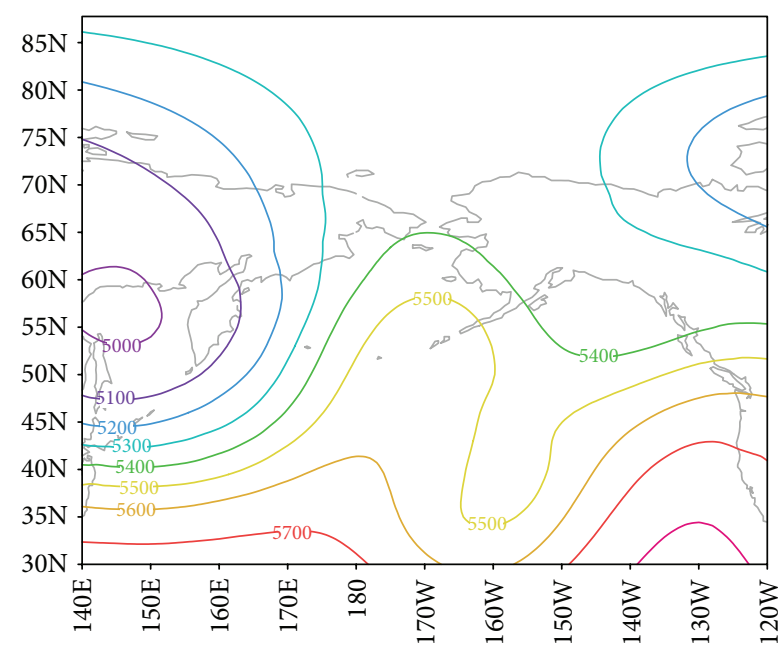

FIGURE 8: GEFS ensemble mean time-averaged geopotential heights for March 9-14, 2013.

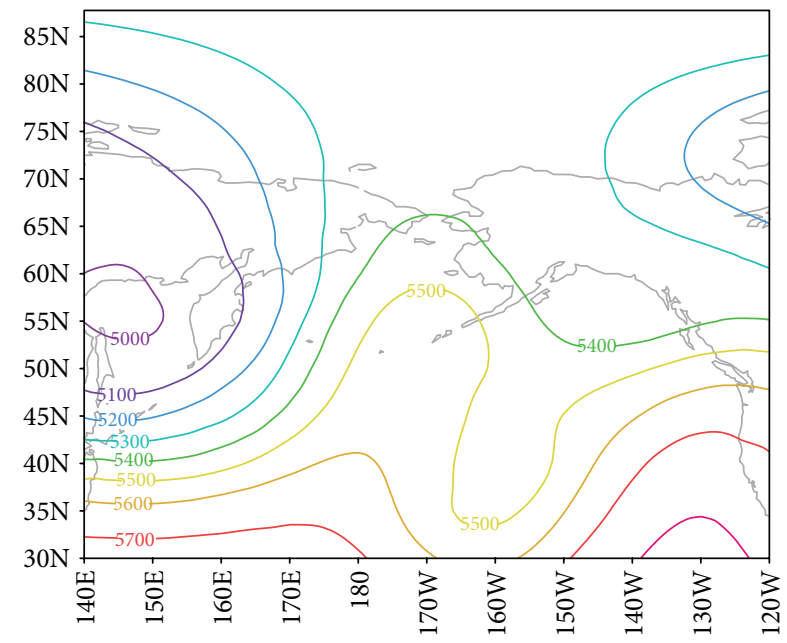

FIGURE 9: GEFS control time-averaged geopotential heights for March 9-14, 2013.

at onset and rise to local maxima by the end of the blocking event.

Now, the DIRE for the GEFS mean, control, and two other ensemble members are plotted alongside the ERA-Interim DIRE for comparison (see Figure 12). For this case, all of the GEFS members plotted show a distinct maximum in instability at block onset as reflected by their crossing the time-axis from positive to negative (M1 crossed before), but none of them crosses at block decay. They all assume positive values indicating increasing instability, but not necessarily a maximum.

\section{Discussion and Conclusions}

For the two blocking events considered here, the higher resolution ERA-Interim reanalysis behaved as expected (and perhaps better than the NCEP-NCAR reanalysis) from previous research (see $[6,7])$. However, the relatively higher 


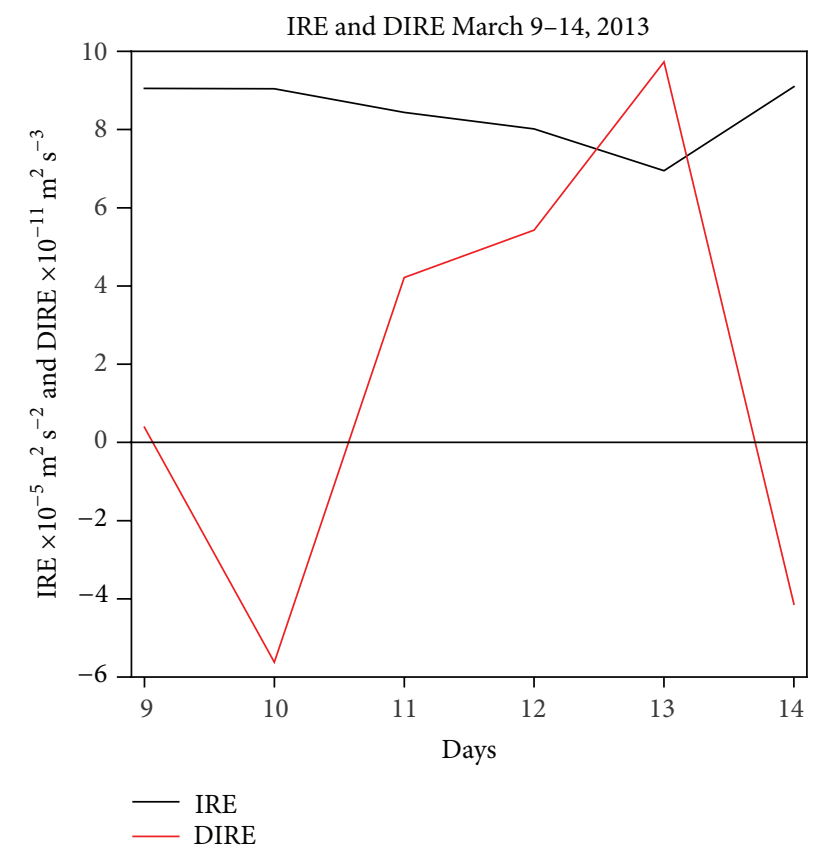

FIGURE 10: IRE and DIRE from ERA-Interim reanalysis for March 9-14, 2013.

resolution than NCEP-NCAR reanalysis, in both cases the GEFS ensemble mean and GEFS control, failed to reproduce the expected behavior of the IRE as in the ERA reanalysis. However, as mentioned above, the outliers in both cases contributed to the poor performance of the ensemble mean in GEFS. The two representative ensemble members behaved much closer to the ERA renalysis and as expected based on previous research. The DIRE, on the other hand, seemed to behave more as expected in GEFS at block onset, but the two representative ensemble members M1 and M2 still appeared to behave more realistically overall than the ensemble mean and control, especially, in October blocking event. Hence, it appears that the behavior of the enstrophy-based diagnostics IRE and DIRE in these two cases is better handled in an ensemble than with a single dynamical control forecast. The ensemble mean in both cases considered here is a rather poor indicator of the behavior of the diagnostics. Here, an ensemble is essential for obtaining the expected results in a weather model. The use of the diagnostics in these cases is considerably improved with an ensemble, since the control does not perform as well as expected. It is likely that plots of several of the members of an ensemble would make the diagnostics more useful for studying blocking events in a weather model. Thus, even though the relatively high resolution in the ERA reanalysis (compared to NCEPNCAR reanalysis) demonstrates the expected behavior of the diagnostics, an ensemble was essential for weather model. It is worth pointing out that using the IRE and DIRE together provides the best estimate of the instability associated with block onset and decay. The DIRE behaved overall in a better way at block onset than decay. It appears that block decay may still be underpredicted as explained in $[9,10]$.

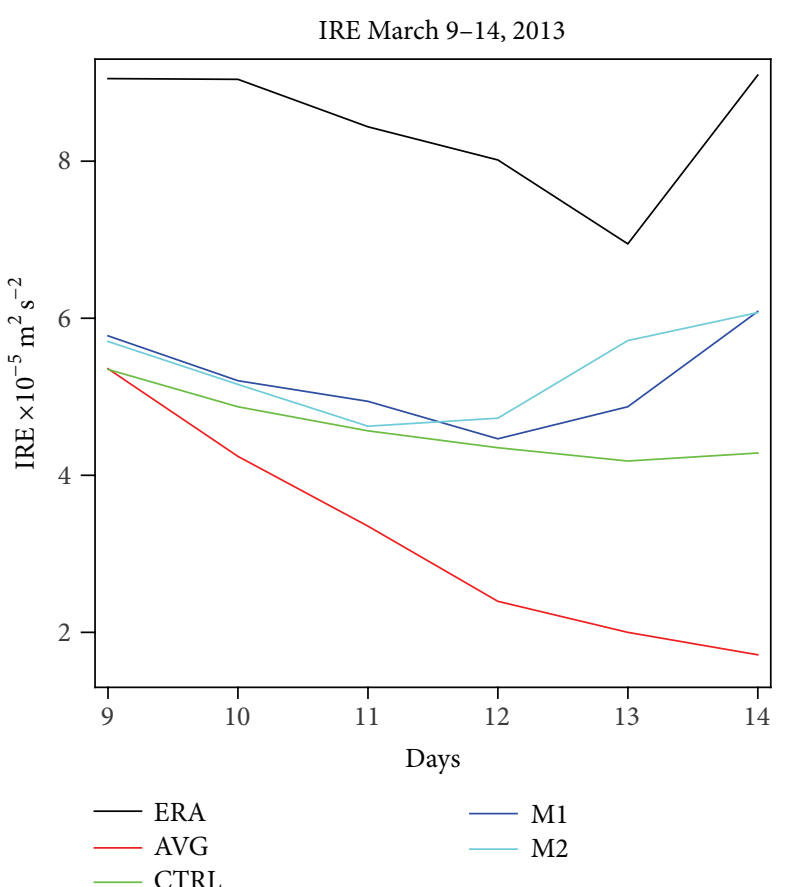

FIGURE 11: ERA and GEFS values of IRE for March 9-14, 2013. Shown are ERA reanalysis (black), GEFS ensemble mean (red), GEFS control (green), and two other ensemble members (dark and light blue).

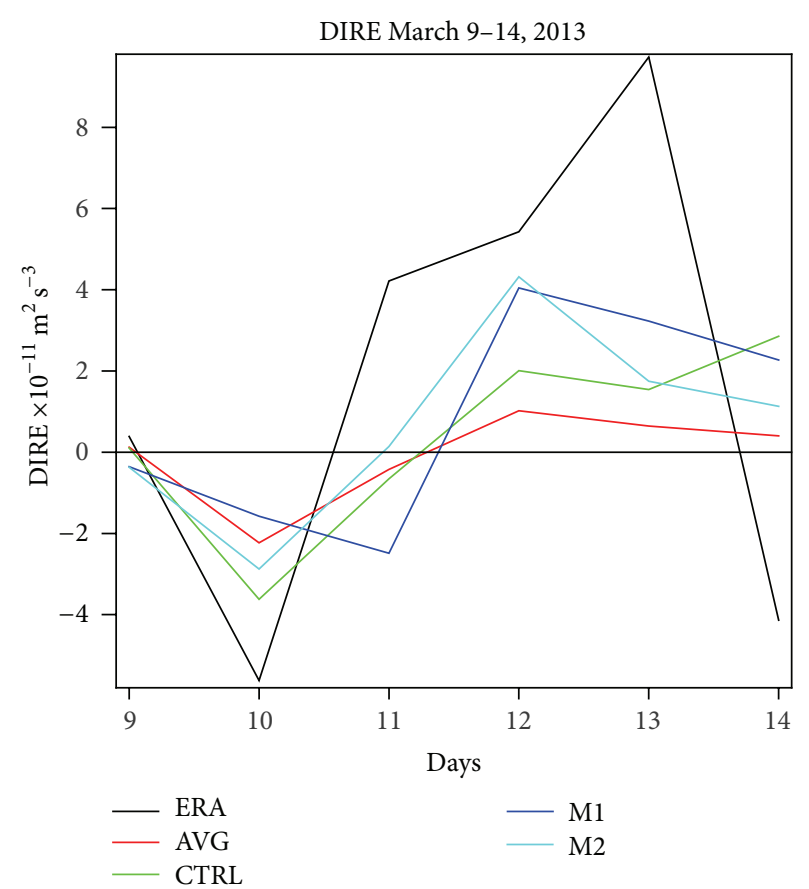

FIGURE 12: ERA and GEFS values of DIRE for March 9-14, 2013. Shown are ERA reanalysis (black), GEFS ensemble mean (red), GEFS control (green), and two other ensemble members (dark and light blue). 
In this paper, the two enstrophy-based diagnostics, the integrated enstrophy (IRE) and its derivative (DIRE) assuming inviscid barotropic flow, have been used to study two blocking events. The ERA-Interim reanalysis and the Global Ensemble Forecast System (GEFS) have been used in the study of these two blocking events. The enstrophy-based diagnostics were found to behave as expected in the ERA reanalysis and required a more subtle analysis in GEFS.

\section{Acknowledgments}

The authors wish to thank the two anonymous reviewers for their insightful and helpful comments, which have considerably improved the clarity and strength of this paper.

\section{References}

[1] A. Fournier, "Atmospheric energetics in the wavelet domain. Part II: time-averaged observed atmospheric blocking," Journal of the Atmospheric Sciences, vol. 60, no. 2, pp. 319-338, 2003.

[2] A. R. Hansen and A. Sutera, "A comparison of the spectral energy and enstrophy budgets of blocking versus nonblocking periods," Tellus A, vol. 36, no. 1, pp. 52-63, 1984.

[3] V. P. Dymnikov, Y. V. Kazantsev, and V. V. Kharin, "Information entropy and local Lyapunov exponents of barotropic atmospheric circulation," Izvestiya, Atmospheric and Oceanic Physics, vol. 28, no. 6, pp. 425-432, 1993.

[4] S. Tibaldi and F. Molteni, "On the operational predictability of blocking," Tellus A, vol. 42, no. 3, pp. 343-365, 1990.

[5] H. Athar and A. R. Lupo, "Scale and stability analysis of blocking events from 2002 to 2004: a case study of an unusually persistent blocking event leading to a heat wave in the Gulf of Alaska during August 2004," Advances in Meteorology, vol. 2010, Article ID 610263, 15 pages, 2010.

[6] A. D. Jensen and A. R. Lupo, "Using enstrophy as a diagnostic to identify blocking regime transition," Quarterly Journal of the Royal Meteorological Society, 2013.

[7] A. R. Lupo, I. I. Mokhov, S. Dostoglou, A. R. Kunz, and J. P. Burkhardt, "Assessment of the impact of the planetary scale on the decay of blocking and the use of phase diagrams and enstrophy as a diagnostic," Izvestiya, Atmospheric and Oceanic Physics, vol. 43, no. 1, pp. 45-51, 2007.

[8] A. R. Lupo, I. I. Mokhov, M. G. Akperov, A. V. Cherokulsky, and H. Athar, "A dynamic analysis of the role of the planetary and synoptic scale in the summer of 2010 blocking episodes over the European part of Russia," Advances in Meteorology, vol. 2012, Article ID 584257, 11 pages, 2012.

[9] J. S. Watson and S. J. Colucci, "Evaluation of ensemble predictions of blocking in the NCEP global spectral model," Monthly Weather Review, vol. 130, no. 12, pp. 3008-3021, 2002.

[10] J. L. Pelly and B. J. Hoskins, "How well does the ECMWF ensemble prediction system predict blocking?" Quarterly Journal of the Royal Meteorological Society, vol. 129, no. 590, pp.1683-1702, 2003.

[11] J. S. Frederiksen, M. A. Collier, and A. B. Watkins, "Ensemble prediction of blocking regime transitions," Tellus A, vol. 56, no. 5, pp. 485-500, 2004.

[12] F. Molteni and T. N. Palmer, "Predictability and finite-time instability of the northern winter circulation," Quarterly Journal, vol. 119, no. 510, pp. 269-298, 1993.
[13] D. Luo, "A barotropic envelope Rossby soliton model for blockeddy interaction. Part I: effect of topography," Journal of the Atmospheric Sciences, vol. 62, no. 1, pp. 5-21, 2005.

[14] A. R. Lupo and P. J. Smith, "Climatological features of blocking anticyclones in the northern Hemisphere," Tellus A, vol. 47, no. 4, pp. 439-456, 1995.

[15] D. F. Rex, "Blocking action in the middle troposphere and its effect on regional climate I: the climatology of blocking action," Tellus, vol. 2, no. 3, pp. 196-211, 1950.

[16] F. Rex, "Blocking action in the middle troposphere and its effect on regional climate II: the climatology of blocking action," Tellus, vol. 3, pp. 275-301, 1950.

[17] H. Lejenas and H. Okland, "Characteristics of northern Hemisphere blocking as determined from a long time series of observational data," Tellus A, vol. 35, no. 5, pp. 350-362, 1983. 

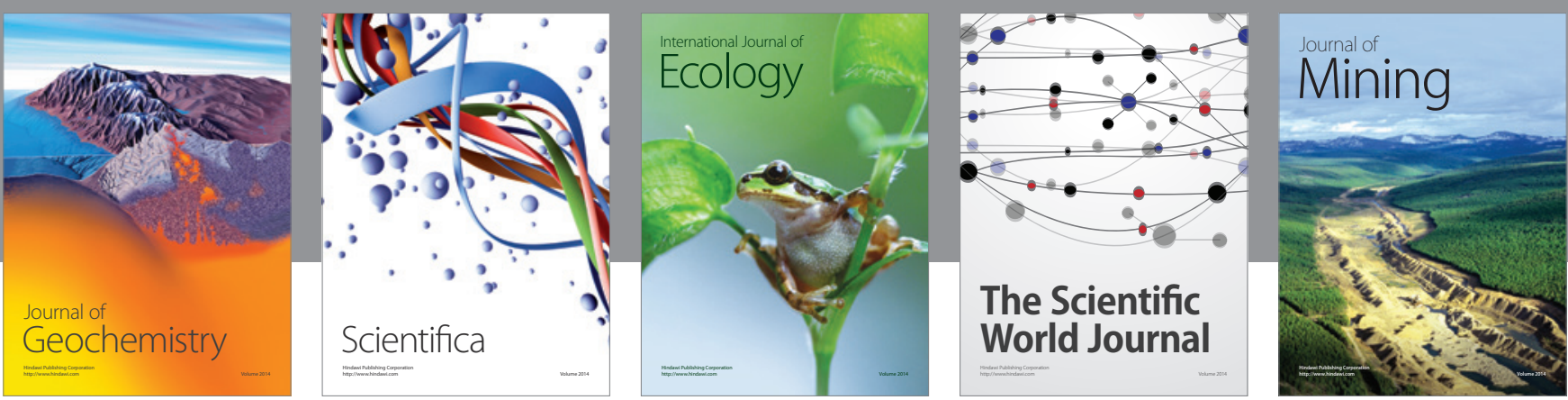

The Scientific World Journal
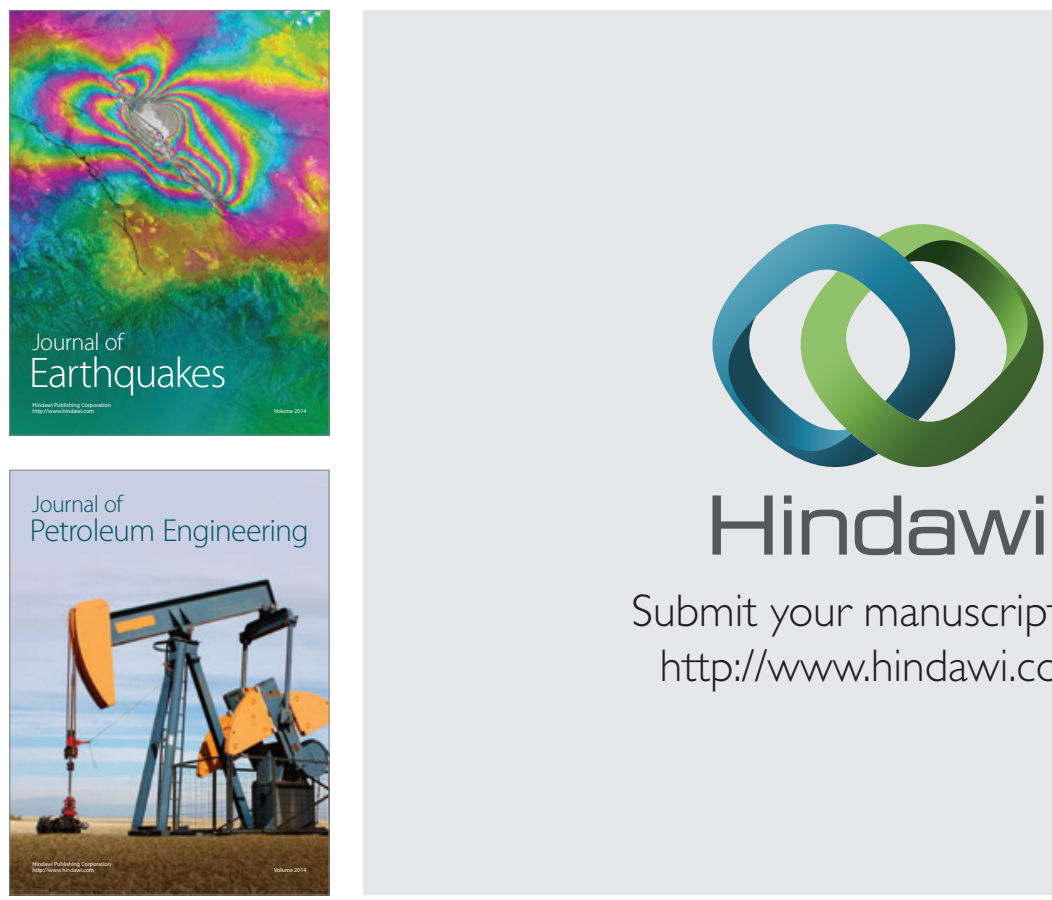

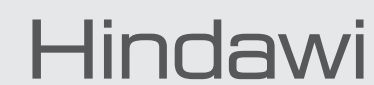

Submit your manuscripts at

http://www.hindawi.com
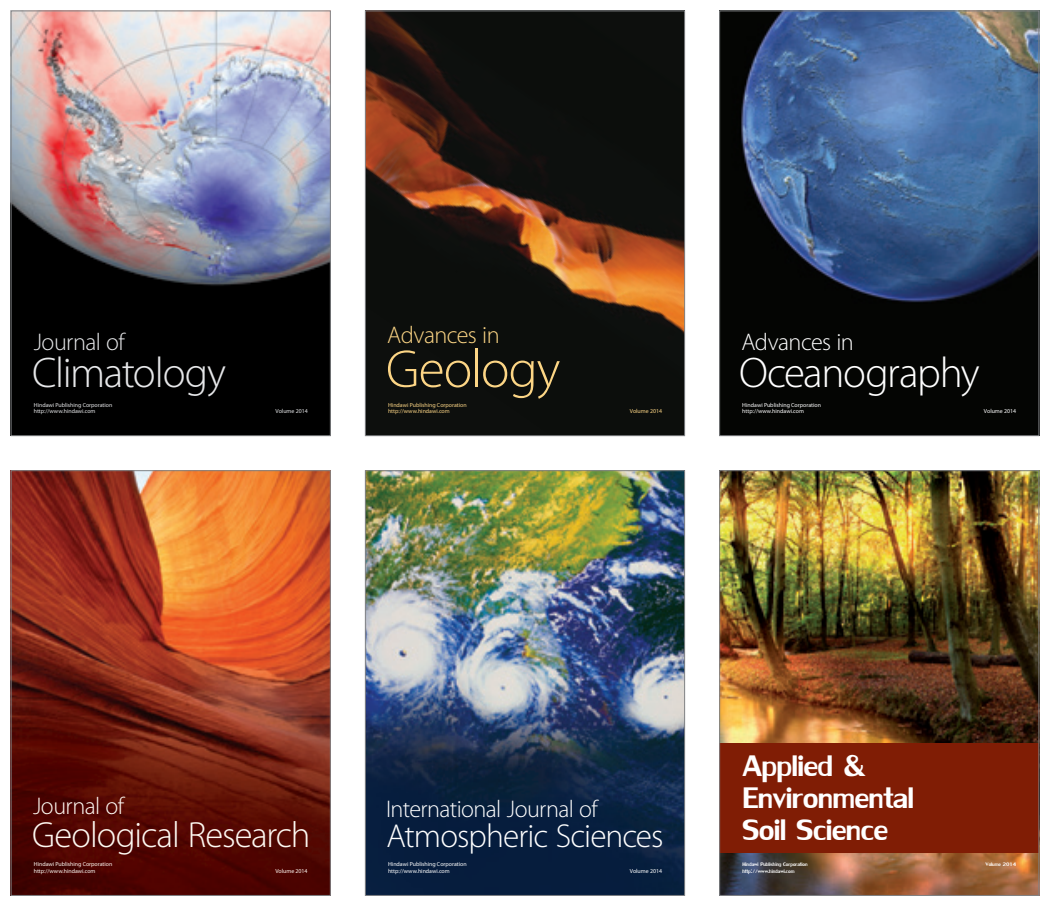
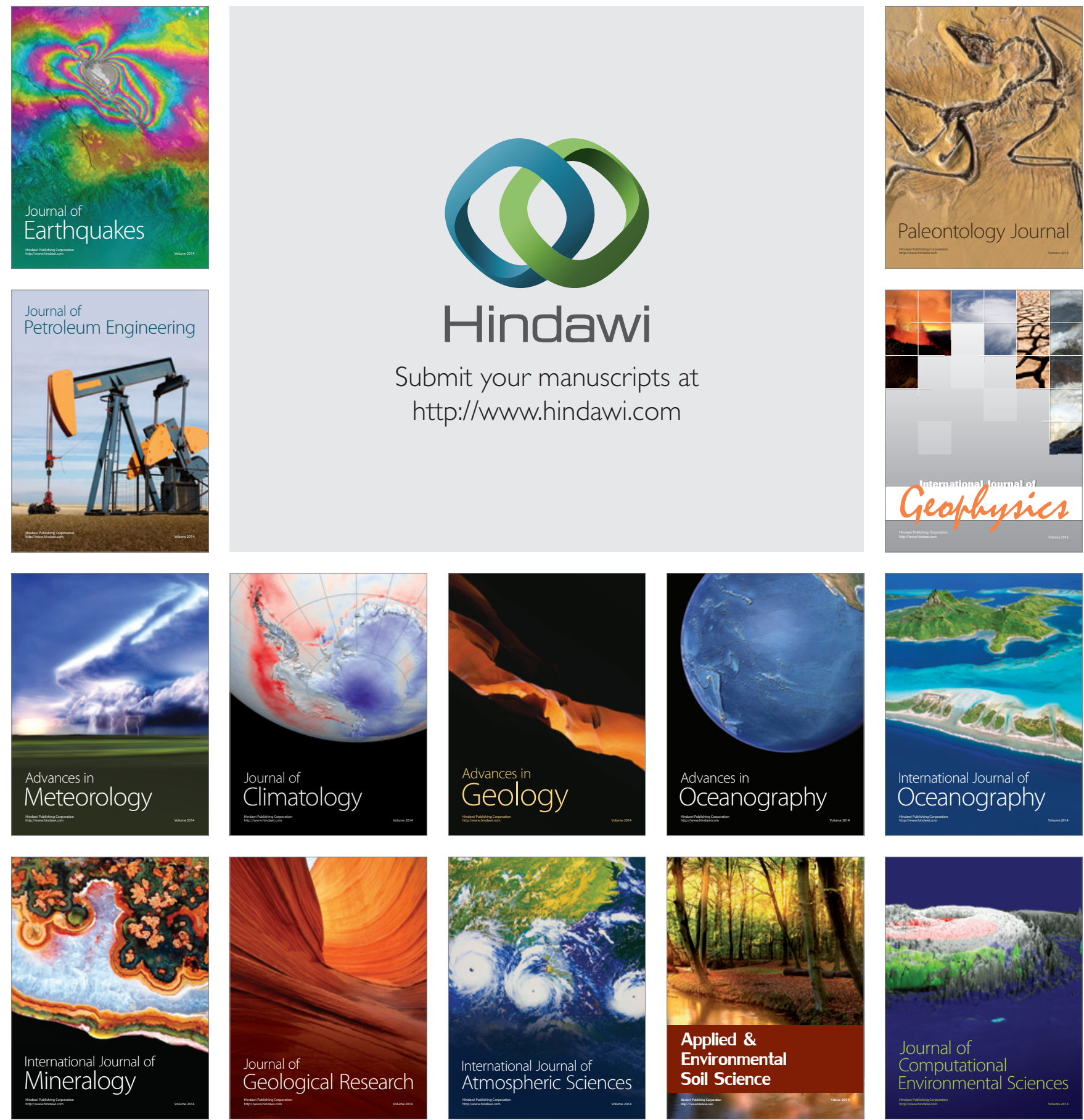